EPJ Web of Conferences 71, 00039 (2014)

DOI: 10.1051/epjconf/20147100039

(C) Owned by the authors, published by EDP Sciences, 2014

\title{
Charm and Beauty in Photoproduction at HERA
}

\author{
Monica Dobre ${ }^{1, \mathrm{a}}$ on behalf of the $\mathrm{H} 1$ and ZEUS Collaborations \\ ${ }^{1}$ National Institute for Physics and Nuclear Engineering Horia Hulubei, Bucharest, Romania
}

\begin{abstract}
The photoproduction of beauty and charm quarks at the ep collider HERA are presented. The b-quarks production was investigated in the $b b \rightarrow e e X^{\prime}$ channel and the differential production cross section was measured as a function of the average transverse momentum of the beauty quarks down to the threshold. The cross section of $\mathrm{D}^{*}$ meson decaying in the golden channel was determined both inclusively and in $\mathrm{D}^{*}$-tagged dijet events. Exploiting the characteristics of the heavy-flavoured hadron decays, beauty and charm quark cross sections were also measured in dijet events using secondary vertices or semi-muonic decays.
\end{abstract}

\section{Introduction}

Measurements of heavy flavour production performed with the H1 and ZEUS detectors in (ep) collisions at HERA give valuable input for stringent tests of the theory of quantum chromodynamics (pQCD) predictions, since the large masses of the charm and beauty quarks provide a hard scale for the perturbative calculations. In leading order (LO), the heavy flavour quarks ${ }^{1}$ are produced mainly through the boson gluon fusion (BGF) process, $\gamma g \rightarrow Q \bar{Q}(Q=c, b)$.

In photoproduction, a quasi-real photon is emitted from the incoming electron and collides with the proton. There are two types of mechanisms contributing: direct, when the photon emitted by the electron ${ }^{2}$ behaves as a point-like particle in the hard process, and resolved, when the photon fluctuates into a hadronic state before the hard interaction, of which one parton participates in the hard interaction. The contribution of the resolved processes is expected to be significant in LO. The results presented here were obained using data from collisions of electrons with an energy of 27.6 $\mathrm{GeV}$ and protons with an energy of $920 \mathrm{GeV}$, thus having an energy of $320 \mathrm{GeV}$ available in the centre-of-mass system.

\section{QCD Models}

The results were compared to LO calculations, obtained using the Pythia [1], Herwig [2] and Cascade [3] Monte Carlo generators. The Pythia and Herwig Monte Carlo generators are making use of the DGLAP [7] evolution whereas Cascade is based on the CCFM [8] evolution. The Monte Carlo generators are also used for estimating the detector acceptance and the reconstruction efficiency.

\footnotetext{
ae-mail: monica.dobre@cern.ch

${ }^{1}$ Hereafter, the term 'heavy' refers only to the $c$ - and $b$-quarks.

${ }^{2}$ In this paper the term 'electron' refers to both electrons and positrons
}

This is an Open Access article distributed under the terms of the Creative Commons Attribution License 2.0, which permits unrestricted use, distribution, and reproduction in any medium, provided the original work is properly cited. 
The results were also compared to the NLO calculations FMNR[4], MC@ NLO [5] and GMVFNS [6]. FMNR and MC@NLO calculations are performed in the fixed flavour number scheme. This scheme assumes that the massive $\mathbf{c}$ and $\mathbf{b}$ quarks are generated dynamically via the boson-gluon fusion, whereas the proton is described by parton density functions of gluons and light-flavoured quarks alone. The calculation is expected to predict mass threshold effects correctly and to be reliable at small scales, of the order of the masses of the heavy quarks. In the GMVFNS calculation the heavy quark is treated as massive at small scales and massless at large scales. This calculation is performed only for the c-quark.

\section{Beauty Photoproduction near Threshold Using Di-electron Events}

In this analysis [9] the differential beauty cross section in photoproduction is measured in the channel $e p \rightarrow e b \bar{b} X$ with the $\mathrm{H} 1$ detector [10], using the semileptonic decays of the heavy flavours into electrons. The events were recorded by identifying electrons with a transverse momentum as low as 1 $\mathrm{GeV}$ using a dedicated online trigger and offline electron identification. The data was recorded in the year 2007 and corresponds to an integrated luminosity of $48.1 \mathrm{pb}^{-1}$.

The background to open beauty production in the selected di-electron sample is dominated by events from inelastic $J / \psi$-meson production. Other contributions include the production of light quarks and of open charm. For the reconstruction of the transverse momentum of the $b$-quarks a hemisphere method [11] is used. For separating the different background sources contributing the di-electron signature and discriminating the $b \bar{b}$ signal against the remaining background, a template method is employed. Independent phase space regions are defined for the production of light quarks, open charm production, the production of $J / \psi$-mesons and the $b$-signal. The contributions for signal and individual background sources are obtained through an unfolding procedure.

The visible cross section is measured for the phase space defined by the virtuality of the photon $Q^{2} \leq 1 \mathrm{GeV}^{2}$, inelasticity $0.05 \leq y \leq 0.65$ and the pseudorapidity of the $b$-quark $|\eta(b)|,|\eta(\bar{b})| \leq 2$. The sources of systematic uncertainties are the electron identification, the track finding efficiency of the electron, the trigger efficiency, the model uncertainty, the charm production, the fragmentation function of the heavy quarks, contributions from the remaining $u d s$ background, DIS background and the simulation of the radiative tail of the $J / \psi \rightarrow$ ee events.

The measured differential beauty cross section is compared in Figure 3 with an NLO QCD prediction in the fixed flavour number scheme as calculated by the program FMNR. The cross section at low transverse momenta has the smallest uncertainties. The prediction tends to underestimate the data, a trend also observed in previous beauty measurements at larger transverse beauty momenta. The inclusive beauty photoproduction cross section is found to be:

$$
\sigma(e p \rightarrow e b \bar{b} X)=3.79 \pm 0.53(\text { stat }) \pm 0.58(\text { syst }) \mathrm{nb},
$$

whereas the NLO prediction obtained from FMNR is $\sigma(e p \rightarrow e b \bar{b} X)=2.40_{-0.49}^{+0.55} \mathrm{nb}$.

\section{Measurement of Inclusive and Dijet $D^{*}$ Meson Cross Sections in Photoproduction at HERA}

In this analysis [12] the inclusive photoproduction of $D^{*}$ mesons and of $D^{*}$-tagged dijets is investigated using the $\mathrm{H} 1$ detector, with the $D^{*}$ meson decaying in the so-called "golden channel": $D^{* \pm} \rightarrow D^{0} \pi_{\text {slow }}^{ \pm} \rightarrow K^{\mp} \pi^{ \pm} \pi_{\text {slow }}^{ \pm}$with a branching ratio of $\mathcal{B R}=2.63 \pm 0.04 \%$ [13]. The data sample was recorded in the years 2006 and 2007 and has a total integrated luminosity of $113 \mathrm{pb}^{-1}$. 
ICNFP 2013

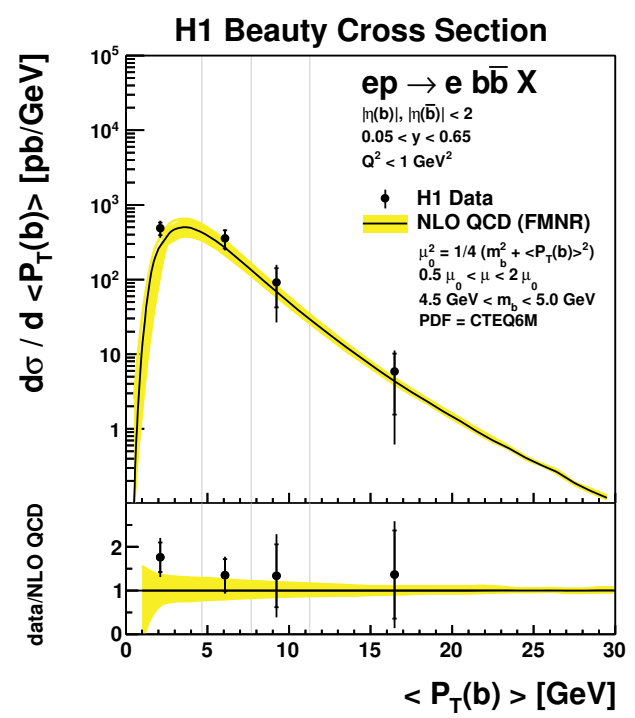

Figure 1. Differential beauty cross section $d \sigma / d<P_{T}(b)>$ shown as a function of the quadratically averaged transverse momentum of the beauty quarks $\left\langle P_{T}(b)\right\rangle$ (upper part). The data are represented by points with the inner vertical error bars representing the statistical errors and the full error bars representing the total uncertainty. The vertical grey lines indicate the bin boundaries in $\left\langle P_{T}(b)\right\rangle$ of each data point and the points are shown at the bin centre positions. The data are compared to the FMNR NLO QCD calculation (solid line) with the uncertainty represented as a shaded band. In the lower part, the ration of the measured cross section to the calculated NLO QCD prediction is shown.

The definitions of the phase space for the two measurements are given in Table 1. The jets are obtained using the inclusive $k_{T}$-algorithm [14] in the energy recombination scheme with jet size $\Delta R=\sqrt{(\Delta \eta)^{2}+(\Delta \phi)^{2}}=1$. The wrong charge combinations, defined as $K^{ \pm} \pi^{ \pm} \pi_{\text {slow }}^{ \pm}$, are used for the estimation of the shape of the combinatorial background in the signal region. The number of reconstructed $D^{*}$ mesons $N\left(D^{*}\right)$ is obtained by a simultaneous log-likelihood fit to the right charge and the wrong charge $\Delta M$ distributions in each analysis bin, where $\Delta M=m\left(K \pi \pi_{\text {slow }}\right)-m(K \pi)$. The asymmetric Crystal Ball function [15] is used for fitting the signal and the Granet function [16] for the background shape parametrisation. The fit to the inclusive $D^{*}$ sample yields $8232 \pm 164 D^{*}$ mesons and the fit to the $D^{*}$-tagged dijet sample yields $3937 \pm 114 D^{*}$ mesons.

The sources of systematic uncertainties are the trigger efficiency, the signal extraction, the $D^{0}$ mass cut, the contribution from decay modes of $D^{0}$ other than the one considered, the DIS contribution, the $d E d x$ cut, the hadronic energy scale, the model, the fragmentation of the charm quark, the track finding efficiency, the luminosity and the branching ratio.

The total visible cross section for the inclusive $D^{*}$ meson photoproduction is measured to be:

$$
\sigma_{v i s}\left(e p \rightarrow e D^{*}+X\right)=41.1 \pm 0.8 \text { (stat.) } \pm 3.6 \text { (syst.) } \pm 2.7 \text { (norm.) } \mathrm{nb}
$$

in the kinematic range defined in Table 1. The LO predictions from PYTHIA and CASCADE amount to $43.7 \mathrm{nb}$ and $32.9 \mathrm{nb}$, respectively. The NLO calculations predict $26_{-8}^{+13} \mathrm{nb}$ for FMNR, $37_{-14}^{+28} \mathrm{nb}$ for GMVFNS and $30_{-7}^{+6} \mathrm{nb}$ for MC@NLO. The cross section as a function of the transverse momentum of the $D^{*}$, the pseudorapidity of the $D^{*}$ and the energy in the photon-proton centre-of-mass system are compared to the NLO predictions and shown in Figure 2. The $D^{*}$ kinematics is well described, as well as $W_{\gamma p}$. 


\begin{tabular}{|c|c|}
\hline \multicolumn{2}{|c|}{ Inclusive $D^{*}$ meson and $D^{*}$-tagged dijet production } \\
\hline Photon virtuality & $Q^{2}<2 \mathrm{GeV}^{2}$ \\
\hline$\gamma p$ centre-of-mass energy & $100<W_{\gamma p}<285 \mathrm{GeV}$ \\
\hline Pseudorapidity of $D^{* \pm}$ & $\left|\eta\left(D^{*}\right)\right|<1.5$ \\
\hline \multicolumn{2}{|c|}{ Inclusive $D^{*}$ meson production } \\
\hline Transverse momentum of $D^{* \pm}$ & $p_{T}\left(D^{*}\right)>1.8 \mathrm{GeV}$ \\
\hline \multicolumn{2}{|c|}{$\bar{D}^{*}$-tagged dijet production } \\
\hline Transverse momentum of $D^{* \pm}$ & $p_{T}\left(D^{*}\right)>2.1 \mathrm{GeV}$ \\
\hline Transverse momentum of $D^{*}$ jet & $p_{T}\left(D^{*}\right.$ jet $)>3.5 \mathrm{GeV}$ \\
\hline Pseudorapidity of $D^{*}$ jet & $\mid \eta\left(D^{*}\right.$ jet $) \mid<1.5$ \\
\hline Transverse momentum of the other jet & $p_{T}($ other jet $)>3.5 \mathrm{GeV}$ \\
\hline Pseudorapidity of other jet & $-1.5<\eta$ (other jet $)<2.9$ \\
\hline Dijet invariant mass $M_{j j}$ & $M_{j j}>6 \mathrm{GeV}$ \\
\hline
\end{tabular}

Table 1. The kinematic range of the measurements
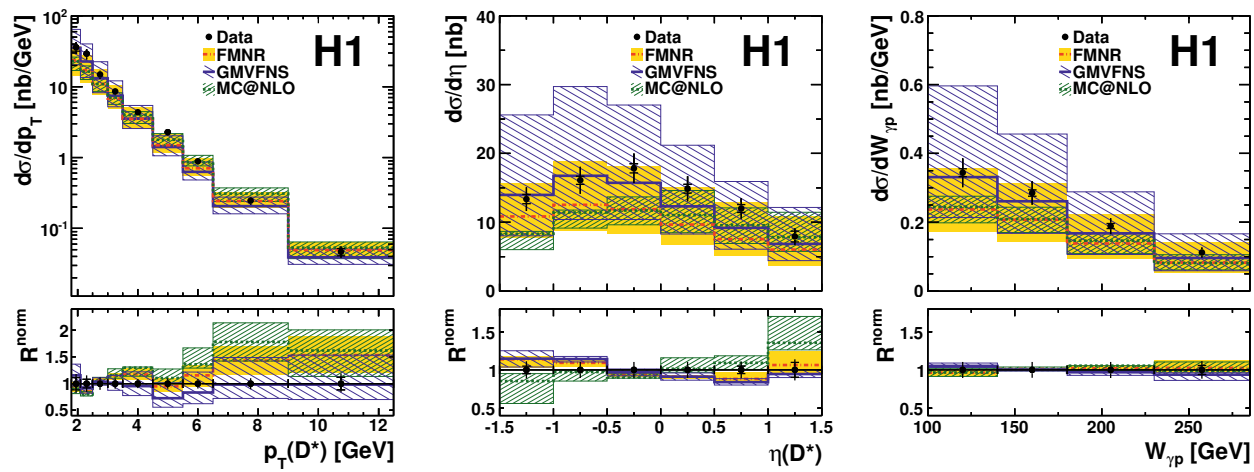

Figure 2. Cross section as a function of $p_{T}\left(D^{*}\right)$ (left), $\eta\left(D^{*}\right)$ (middle) and $W_{\gamma p}$ (right). The data is represented by black points and the NLO predictions by coloured bands. The vertical error bars represent the total uncertainty, with the inner error bars depicting the statistical uncertainty.

The integrated visible $D^{*}$-tagged dijet cross section is measured to be:

$$
\left.\sigma_{v i s}\left(e p \rightarrow e D^{*} \text { jet }+ \text { other jet }+X\right)=9.68 \pm 0.28 \text { (stat.) } \pm 0.51 \text { (syst. }\right) \pm 0.64 \text { (norm.) nb }
$$

in the kinematic range defined in Table 1. The predictions from PYTHIA, CASCADE and MC@NLO amount to $8.9 \mathrm{nb}, 8.1 \mathrm{nb}$ and $7.1_{-1.8}^{+2.5} \mathrm{nb}$, respectively. In the common range of transverse momentum, $p_{T}\left(D^{*}\right)>2.1 \mathrm{GeV}$, the ratio of the $D^{*}$-tagged dijet to the inclusive $D^{*}$ cross section is $0.304 \pm 0.013 \pm$ 0.031, compared to 0.271 for PYTHIA, 0.311 for CASCADE and $0.309_{-0.040}^{+0.019}$ for MC@ NLO. In order to further investigate the charm production dynamics, variables related to the hadronic final state are investigated. Two of these variables are the difference in the azimuthal angle between the two jets, $\Delta \phi$, and the fraction of the longitudinal photon momentum entering the hard scattering process, $x_{\gamma}=\frac{\sum_{j e t s}\left(E-p_{z}\right)_{i}}{\sum_{H F S}\left(E-p_{z}\right)_{j}}$. The cross section as a function of the transverse momentum of the $D^{*}$ jet, the azimuthal angular differences between the two jets and $x_{\gamma}$ are compared to the NLO predictions and shown in Figure 3. The MC@ NLO underestimates the data in the low jet transverse momenta region, resulting in a smaller visible cross section. Also, some difference in shape are observed in the $x_{\gamma}$ distribution. 

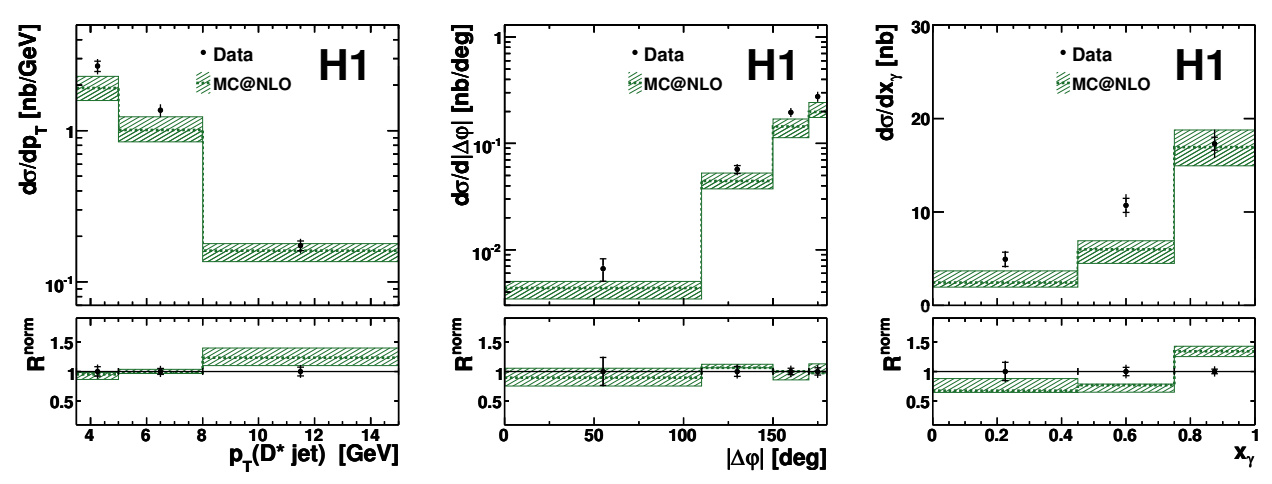

Figure 3. Cross section as a function of $p_{T}\left(D^{*}\right.$ jet) (left), $\Delta \phi$ (middle) and $x_{\gamma}$ (right). The data is represented by black points and the NLO predictions by coloured bands. The vertical error bars represent the total uncertainty, with the inner error bars depicting the statistical uncertainty.

\section{Beauty and Charm in Dijet Events with Semi-muonic Decays}

In this analysis [17], beauty and charm events with dijets and a muon are selected, the muon being associated with one of the jets. The data were collected in the years 2006 and 2007 and correspond to an integrated luminosity of $179 \mathrm{pb}^{-} 1$. The main kinematic cuts are on the photon virtuality: $Q^{2}<2.5$ $\mathrm{GeV}^{2}$, inelasticity: $0.2<y<0.8$, muon kinematics: $p_{T}(\mu)>2.5 \mathrm{GeV}^{2}$ and $-1.3<\eta(\mu)<1.5$, and on the jet kinematics: $p_{T}($ jet $(1,2))>7(6) \mathrm{GeV}$ and $-1.5<\eta($ jet $)<2.5$. The jets are reconstructed using the inclusive $k_{T}$-algorithm in the massless $P_{T}$ recombination scheme and with the distance parameter $R_{C}=1$ in the $\eta-\phi$ plane $[14,18]$. The separation of $b, c$ and light quark (uds) events is performed using the properties of the muon track associated to the $\mu$-jet. Two variables are defined: the impact parameter $\delta$ of the muon track and the relative transvers momentum of the muon relative to the $\mu$ jet axis, $p_{T}^{\text {rel }}$. The fractions of events with beauty, charm and light quarks are obtained by a binned likelihood fit [19] in the $\delta-p_{T}^{\text {rel }}$ plane.

The sources for systematic uncertainties are the trigger efficiencies, the muon identification efficiency, the track efficiency, the integrated luminosity, the resolution of the $\delta$ parameter, the resolution of the jet azimuthal angle, the hadronic energy scale, the physics model, the parton fragmentation function and the fake muon background. The total visible cross sections for beauty and charm are shown in the Table 2. Charm and beauty cross sections as a function of $x_{\gamma}$, as defined in the previous section, are shown in Figure 4. The charm cross section as a function of $x_{\gamma}$ is overestimated by PYTHIA in the direct-enriched region $\left(x_{\gamma}>0.75\right)$, but reasonably well described by the other models. The beauty cross section as a function of $x_{\gamma}$ is well described in shape by all models and agreement is reached within $2 \sigma$.

\section{Heavy Quark Jet Production}

In this analysis [20], the large masses and the large lifetimes of the heavy flavoured hadrons are exploited. The measurement is performed in the phase space given by $Q^{2}<1 \mathrm{GeV}^{2}$, inelasticity $0.2<y<0.8$ and the jet kinematics: $p_{T}($ jet $(1,2))>7(6) \mathrm{GeV}$ and $\mid \eta($ jet $) \mid<2.5$. The dataset recorded corresponds to an integrated luminosity of $133 \mathrm{pb}^{-1}$. The decay-length significance is defined $S=$ $d / \delta d$, where the decay length $d$ is the distance in the $X-Y$ plane between the interaction point and the 


\begin{tabular}{|c|c|c|}
\hline & $\sigma_{\text {vis }}\left(e p \rightarrow e b \bar{b} X \rightarrow e j j \mu X^{\prime}\right)[\mathrm{pb}]$ & $\sigma_{\text {vis }}(e p \rightarrow e c \bar{c} X 11 \rightarrow e j j \mu X \prime)[\mathrm{pb}]$ \\
\hline \hline H1 Data & $43.3 \pm 2.1($ stat. $) \pm 4.5$ (syst. $)$ & $81.3 \pm 4.3$ (stat. $) \pm 8.5$ (syst.) \\
\hline PYTHIA & 35.3 & 94.3 \\
CASCADE & 29.0 & 76.8 \\
HERWIG & 20.6 & 58.5 \\
MC@NLO & $33.4_{-9.2}^{+7.1}$ & $58.6_{-11.2}^{+29.5}$ \\
\hline
\end{tabular}

Table 2. Total visible measured beauty and charm cross sections together with the statistical and systematic uncertainties. The total predictions from PYTHIA, CASCADE, HERWIG and MC@NLO are given, for the last one the theoretical uncertainties also quoted.
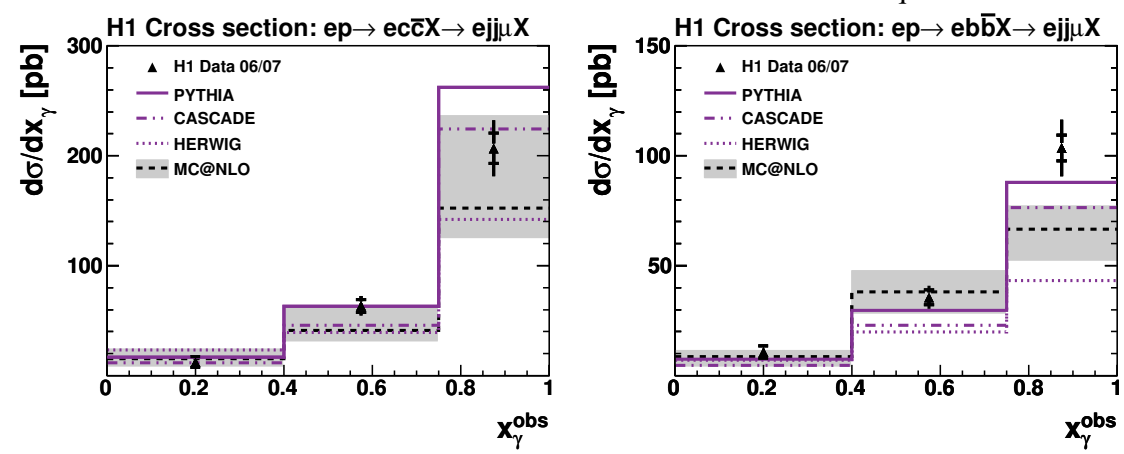

Figure 4. Charm (left) and beauty (right) cross section as a function of $x_{\gamma}$. The data is represented by black points and the NLO predictions by coloured bands. The vertical error bars represent the total uncertainty, with the inner error bars depicting the statistical uncertainty.

secondary vertex, projected onto the jet axis in the $X-Y$ plane. The charm and beauty contributions are obtained by fitting $S$ in three vertex mass bins.

The total beauty and charm cross sections were measured to be:

$$
\sigma_{b}^{v i s}=682 \pm 21 \text { (stat.) } \pm 52 \text { (stat.) pb, } \sigma_{c}^{v i s}=5780 \pm 120 \text { (stat.) } \pm_{-410}^{+390} \text { (stat.) pb }
$$

The FMNR prediction is $740_{-130}^{+210} \mathrm{pb}$ for beauty and $6000_{-1300}^{+2400} \mathrm{pb}$ for charm. The cross sections as a functions of the transverse momentum of the $b$-jet and as a function of the $c$-jet are shown in Figure 5. The NLO calculation is able to describe the data well. Results obtained in several measurements are consistent with one another.

\section{Charm Fragmentation Fractions}

In this analysis [21], the photoproduction of $D^{0}, D^{*+}, D^{+}, D_{s}^{+}$and $\Lambda_{c}^{+}$is studied and the fractions of charm quarks hadronizing into each of them determined. The charm hadrons were reconstructed in the range of transverse momentum $p_{T}>3.8 \mathrm{GeV}$ and pseudorapidity $|\eta|<1.6$. The dataset was recorded between the year 2004 and 2007 and corresponds to an integrated luminosity of $372 \mathrm{pb}^{-1}$. The events were selected in the interval $130<W_{\gamma p}<300 \mathrm{GeV}$.

The sources for systematic uncertainties are: beauty subtraction, rate of the charm-strange baryons, signal extraction, reflections in the mass spectra, the background parametrisation, model dependence, trigger efficiency, track-finding efficiency, hadronic energy scale and resolution. The measured charm fragmentation fraction are summarized in table on the right side of Figure 6 and 

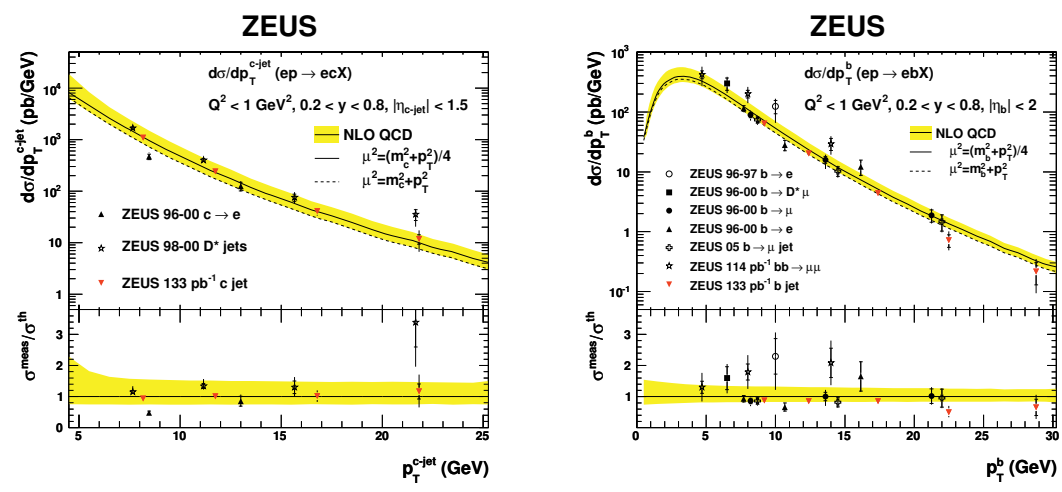

Figure 5. Charm (left) and beauty (right) cross section as a function of the $c$-jet and of the $b$-jet, respectively. The data is represented by black points and the NLO predictions by coloured bands. The vertical error bars represent the total uncertainty, with the inner error bars depicting the statistical uncertainty.

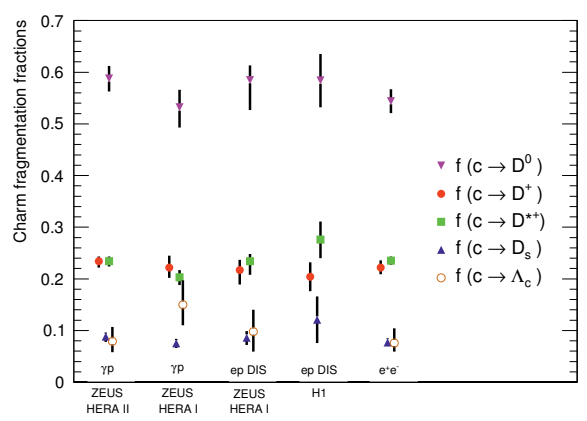

\begin{tabular}{|c|c|c|c|c|}
\hline & \multicolumn{4}{|c|}{ ZEUS $(\gamma p)$ HERA II } \\
\hline & & stat. & syst & br. \\
\hline$f\left(c \rightarrow D^{+}\right)$ & $0.234 \pm$ & 0.006 & & \\
\hline$f\left(c \rightarrow D^{0}\right)$ & $0.588 \pm$ & 0.017 & & \\
\hline$f\left(c \rightarrow D_{s}^{+}\right)$ & $0.088 \pm$ & 0.006 & & \\
\hline$f\left(c \rightarrow \Lambda_{c}^{+}\right)$ & $0.079 \pm$ & 0.013 & & \\
\hline$f\left(c \rightarrow D^{*+}\right)$ & $0.234 \pm$ & 0.006 & $\begin{array}{l}\frac{1.004}{+0.004} \\
-0.004\end{array}$ & $\begin{array}{l}\frac{10.05}{+0.005} \\
-0.007\end{array}$ \\
\hline
\end{tabular}

Figure 6. The charm fragmentation fractions in $e p$ and $e^{+} e^{-}$collisions (left) and their values as measured by the ZEUS experiment (right).

compared with the results from LEP and H1 in Figure 6 (left). The precision of the fragmentation fractions obtained is competitive with measurements performed in $e^{+} e^{-}$collisions. The results from $e p$ and $e^{+} e^{-}$collisions are in agreement with each other, supporting the hypothesis of the universality of the heavy quark fragmentation.

\section{Conclusions}

Several methods are available for measuring the heavy flavour photoproduction in ep collisions. These were used to test the NLO QCD predictions to high precision across multiple scales. In general, good agreement is observed between data and theory, the uncertainties on the measurements already being smaller than the theoretical ones. The fragmentation fractions of the charm quarks measured at HERA are similar to the ones measured at LEP, confirming the universality of the fragmentation fractions. 


\section{References}

[1] T. Sjøstrand et al., PYTHIA 6.1, Comp. Phys. Commun. 135 (2001) 238 [hep-ph/0010017].

[2] G. Corcella et al., JHEP 0101 (2001) 010 [hep-ph/0011363].

[3] H. Jung et al., CASCADE 2.2, Eur. Phys. J. C70 (2010) 1237 [arXiv: 1008.0152].

[4] S. Frixione, P. Nason and G. Ridolfi, Nucl. Phys. B454 (1995) 3 [hep-ph/9506226];

S. Frixione, M.L. Mangano, P. Mason and G. Ridolfi, Phys. Lett. B348 (1995) 633 [hepex/9412348].

[5] S. Frixione and B.R. Webber, JHEP 0206 (2002) 029 [hep-ph/0204244];

S. Frixione, P. Nason and B.R. Webber, JHEP 0308 (2003) 007 [hep-ph/0305252];

T. Toll and S. Frixione, Phys. Lett. B703 (2011) 452 [arXiv: 1106.1614].

[6] G. Kramer and H. Spiesberger, Eur. Phys. J. C38 (2004) 309 [hep-ph/0311062];

B. A. Kniehl, G. Kramer, I. Schienbein and H. Spiesberger, Eur. Phys. J. C62 (2009) 365 [arXiv: 0902.3166].

[7] V.N. Gribov and L.N. Lipatov, Sov. J. Nucl. Phys, 15 (1972) 438 [Yad. Fiz. 15 (1972) 781];

V.N. Gribov and L.N. Lipatov, Sov. J. Nucl. Phys, 15 (1972) 675 [Yad. Fiz. 15 (1972) 1218];

L.N. Lipatov, Sov. J. Nucl. Phys. 20 (1975) 94 [Yad. Fiz. 20 (1972) 181];

Y.L. Dokshitzer, Sov. Phys. JETP 46 (1977) 641 [Zh. Eksp. Teor. Fiz. 73 (1977) 1216];

G. Altarelli and G. Parisi, Nucl. Phys. B126 (1977) 298.

[8] M. Ciafaloni, Nucl. Phys. B296 (1988) 49;

S. Catani, F. Fiorani and G. Marchesini, Phys. Lett. B234 (1990) 339;

S. Catani, F. Fiorani and G. Marchesini, Nucl. Phys. B336 (1990) 18;

G. Marchesini, Nucl. Phys. B445 (1995) 49 [hep-ph/9412327].

[9] F.D. Aaron et al. [H1 Collaboration], Eur. Phys. J. C72 (2012) 2148 [arXiv: 1206. 4346].

[10] I. Abt et al. [H1 Collaboration], Nucl. Instr. Meth. A386 (1997) 310;

I. Abt et al. [H1 Collaboration], Nucl. Instr. Meth. A386 (1997) 348.

[11] M. Sauter, Measurement of Beauty Photoproduction at Threshold Using Di-Electron Events with the H1 Detector at HERA, Ph.D. thesis, ETH Zürich (2009), Diss. ETH No. 18652 and DESYTHESIS-2009-047.

[12] F.D. Aaron et al. [H1 Collaboration], Eur. Phys. J. C72 (2012) 1995, [arXiv: 1203.1170].

[13] K. Nakamura et al. [Particle Data Group], J. Phys. G37 (2010) 075021.

[14] S.D. Ellis and D.E. Soper, Phys. Rev. D48 (1993) 3160 [hep-ph/9305266].

[15] J.E. Gaiser, Charmonium Spectroscopy from Radiative Decays of the $J \psi$ and $\psi^{\prime}, \mathrm{Ph} . \mathrm{D}$. thesis, Stanford University (1982).

[16] P. Granet et al. [French-Soviet Collaboration], Nucl. Phys. B140 (1978) 389.

[17] F.D. Aaron et al. [H1 Collaboration], Eur. Phys. J. C72 (2012) 2047 [arXiv: 1205.2455].

[18] S. Catani, Y.L. Dokshitzer, M.H. Seymour and B.R. Webber, Nucl. Phys. B406 (1993) 187.

[19] R.J. Barlow and C. Beeston, Comput. Phys. Commun. 77 (1993) 219.

[20] H. Abramowicz et al. [ZEUS Collaboration], Eur. Phys. J. C71 (2011) 1659 [arXiv: 1104.5444].

[21] H. Abramowicz et al. [ZEUS Collaboration], JHEP 1309 (2013) 058 [arXiv: 1306.4862]. 